\title{
Expression analysis of Rab11 during zebrafish embryonic development
}

\author{
Haijun Zhang ${ }^{1 \dagger}, Y u \mathrm{Gao}^{1 \dagger}$, Peipei Qian ${ }^{1 \dagger}$, Zhangji Dong ${ }^{1}$, Wenjin $\mathrm{HaO}^{2}$, Dong Liu² and Xuchu Duan ${ }^{2^{*}}$ (D)
}

\begin{abstract}
Background: Rab proteins are GTPases responsible for intracellular vesicular trafficking regulation. Rab11 proteins, members of the Rab GTPase family, are known to regulate vesicular recycling during embryonic development. In zebrafish, there are 3 rab11 paralogues, known as rab11a, rab11ba and rab11bb, sharing high identity with each other. However, the expression analysis of rab11 is so far lacking.

Results: Here, by phylogeny analysis, we found the three rab11 genes are highly conserved especially for their GTPase domains. We examined the expression patterns of rab11a, rab11ba and rab11bb using RT-PCR and in situ hybridization. We found that all the three genes were highly enriched in the central nervous system, but in different areas of the brain. Apart from brain, rab11a was also expressed in caudal vein, pronephric duct, proctodeum, pharyngeal arches and digestive duct, rab11ba was detected to express in muscle, and rab11bb was expressed in kidney, fin and spinal cord. Different from rab11a and rab11ba, which both have maternal expressions in embryos, rab11bb only expresses during $24 \mathrm{hpf}$ to $96 \mathrm{hpf}$.
\end{abstract}

Conclusions: Our results suggest that rab11 genes play important but distinct roles in the development of the nervous system in zebrafish. The findings could provide new evidences for better understanding the functions of rab11 in the development of zebrafish embryos.

Keywords: rab11, Zebrafish, Expression

\section{Background}

Rab/Ypt proteins are small GTP-binding proteins of the Ras superfamily and are key players in intracellular vesicular trafficking regulation [1, 2]. RAB11 was first found from cultured MDCK cell cDNA library screening for homologous genes of yeast YPT1/SEC4 [3], and later identified as the 11th member of the Rab family [4]. Among various Rab proteins, Rab11 is known to localize to recycling endosome and regulate vesicular recycling and primary ciliogenesis, playing a key role in Kupffer vesicle development in zebrafish [5-7].

The Rab11 family is composed of three members of GTPases, Rab11a, Rab11b and Rab25, based on specific motifs [8]. Murine Rab11a and Rab11b were found to localize in distinct cellular compartment, suggesting functional differentiation which have been further proved by

\footnotetext{
* Correspondence: dxd2002sk@ntu.edu.cn

${ }^{+}$Haijun Zhang, Yu Gao and Peipei Qian contributed equally to this work.

${ }^{2}$ College of life science, Nantong University, 9\# Seyuan Road, Nantong

226001, China

Full list of author information is available at the end of the article
}

several recent studies [9]. For instances, Rab11b but rather Rab11a was found to be responsible for endosomal recycling of fibroblast growth factor receptor 4 (FGFR4), a signaling receptor for maintaining tissue homeostasis [10]. In Hela cells, Rab11b but not Rab11a is essentially required for recycling of protease-activated receptor-1 (PAR1), however, depletion of Rab11a could disrupt lysosomal degradation of PAR1 and therefore enhance its intracellular accumulation [11]. Haugsten et al. have just reported that both Rab11a and Rab11b contribute to maintain the endosomal-lysosomal pathway where Rab11a plays the major roles [12]. Although Rab11a subcellular localization has been demonstrated by transgenic zebrafish [13], and molecular function of Rab11 proteins in primary cilia membrane assembly in Kupffer Vesicle has been studied [7], the developmental expression profile of Rab11 proteins in zebrafish remains elusive. In the present project, we showed that zebrafish rab11 genes are conserved in vertebrate evolution by in silicon analysis. We then analyzed the temporal and spatial expression of rab11 genes in embryonic development by RT-PCR and whole mount 
in situ hybridization. Our study provides new insight into the rab11 expression and promotes the use of this model organism to tackle future studies on the role of rab11 in embryo development.

\section{Results}

rab11 genes are highly conserved in vertebrates

We first examined Rab11 protein phylogeny in zebrafish [Rab-11A (NP_001007360); Rab-11BA (NP_999935); Rab11BB (NP_001002555)] and other representative organisms including worm [Caenorhabditis elegans, RAB-11.1 (NP_490675); RAB-11.2 (NP_001251691)], fruit fly [Drosophila melanogaster, Rab11 isoform A (NP_599137); Rab11 isoform B (NP_477170)], medaka [Oryzias latipes, Rab-11A (XP_004086096); Rab-11B (XP_004068250); Rab-11B-like (XP_004068248)], frog [Xenopus tropicalis, Rab-11A (NP_001016481); Rab-11B.1 (NP_001120303)], chicken [Gallus gallus, Rab-11A (NP_001005827); Rab11B (NP_001012569)], mouse [Mus musculus, Rab-11A (NP_059078); Rab-11B (NP_033023)], rat [Rattus norvegicus, Rab-11A (NP_112414); Rab-11B (NP_116006)] and human [Homo sapiens, Rab-11A isoform 1 (NP_004654); Rab-11B (NP_004209)].

The zebrafish Rab11a, Rab11ba and Rab11bb proteins are highly conserved during evolution, especially the GTPase domain, suggesting their important functions. While these paralogues differ in the C-terminal part adjacent to the GTPase domain (Fig. 1a). Zebrafish rab11a was clustered in the rab11a group, and rab11ba and $r a b 11 b b$ in the Rab11b group (Fig. 1b) in the phylogenic tree constructed according to amino acid sequences of all the Rab11 proteins above.

\section{Expression of rab11 genes analyzed by RT-PCR}

We examined rab11a expression using RT-PCR. High expression level of maternal rab11a was detected in both 1-cell stage fertilized eggs and cleavage stages (Fig. 2). Rab11a exhibited steady expression through $96 \mathrm{hpf}$ with a slight lower level found at $12 \mathrm{hpf}$. In adult zebrafish, rab11a was found highly expressed in the eye, fin, brain, eggs and muscle. Its expression in the nervous system is much restricted to the brain, being very weak in the spinal cord. With much similarity to rab11a, rab11ba is steadily expressed from 1-cell stage through 96 hpf, except for a slightly low expression period around $12 \mathrm{hpf}$. Like rab11a, rab11ba is maternally expressed, as detected at 1-cell stage and in the eggs. Rab11ba is also highly expressed in the brain, eggs and muscle and is very weak in the spinal cord. As a paralogue, rab11bb was expressed differently. Rab11bb only showed extremely weak maternal mRNA expression. High zygotic rab11bb expression was not observed until 24 hpf and remained through $96 \mathrm{hpf}$ Rab11bb was detected at high level in kidney, eye, fin and brain, and weakly expressed in muscle. Unlike rab11a and rab11ba, rab11bb expression level in the spinal cord is high.

\section{Rab11a expression during zebrafish embryonic development}

We then performed in situ hybridization to further study the spatial-temporal expression pattern of rab11a as well as rab11ba and rab11bb (Figs. 3, 4, 5). High level of maternal rab11a mRNA was detected at 2 hpf (64-cell) (Fig. 3A). At 12 hpf, zygotic rab11a mRNA was found in the whole organism without tissue specificity (Fig. 3B). As seen at $20 \mathrm{hpf}$ to $24 \mathrm{hpf}$, rab11a expression began to decrease in the trunk and was mainly restricted to the brain and head structures, and also in the caudal spinal cord, caudal vein, pronephric duct and the proctodeum (Fig. 3C-D'). The mRNA expression in the brain remained strong until $36 \mathrm{hpf}$ and decreased in other tissues (Fig. 3E). From $48 \mathrm{hpf}$ to $72 \mathrm{hpf}$, rab11a mRNA was expressed mainly in the brain, with expression in the posterior hindbrain becoming weaker (Fig. 3F, G). At 48 hpf, rab11a expression was also confirmed to express in the brain especially the cerebral cortex by histological analysis of sectioned embryos (Fig. 3I-I"). At 4 dpf, rab11a was strongly expressed in the brain and also in the pharyngeal arches and the digestive duct (Fig. 3H-H").

\section{Rab11ba expression during zebrafish embryonic development}

Similar to rab11a, maternal rab11ba mRNA was detected at $3 \mathrm{hpf}$ (1 k-cell) (Fig. 4A). At $16 \mathrm{hpf}$, rab11ba was expressed in the whole organism and especially in the neural tube (Fig. 4B-B'). At $20 \mathrm{hpf}$, rab11ba expression in the brain remained strong, but its mRNA was not detected in the spinal cord (Fig. 4C, C'). Like that of rab11a, rab11ba mRNA was seen in the pronephric duct and proctodeum (Fig. 4C). At $24 \mathrm{hpf}$, rab11ba was maintained in the brain (Fig. 4D). At this stage, rab11ba started its expression in the pectoral fins. Rab11ba expression in the spinal cord and the caudal vein was significantly decreased at $36 \mathrm{hpf}$ and again increased at $48 \mathrm{hpf}$ (Fig. 4E-F). At this stage, rab11ba was expressed at proctodeum at $48 \mathrm{hpf}$. Transverse section analysis demonstrated almost the same expression patterns of Rab11ba as that of Rab11a where it was expressed in the brain at $48 \mathrm{hpf}$ as well (Fig. 4I-I"). From $72 \mathrm{hpf}$ to $96 \mathrm{hpf}$, rab11ba was expressed in the brain, retina, olfactory placode and pectoral fins (Fig. 4G$\left.H^{\prime}\right)$. Rab11ba was also found highly expressed in craniofacial neuromasts at $72 \mathrm{hpf}$ and $96 \mathrm{hpf}$, which was unique compared to rab11a and rab11bb (Fig. 4G-H').

\section{Rab11bb expression during zebrafish embryonic development}

No maternal message was detected of rab11bb at $2 \mathrm{hpf}$ (64-cell) (Fig. 5A). Rab11bb expression was seen in the 


\section{A}

Rab-11.1_C.elegans Rab-11.2_C.elegans Rab-11_fruitfly Rab-11a medaka Rab-11b medaka Rab-11b'_medaka Rab-11a_zebrafish Rab-11ba_zebrafish Rab-11bb_zebrafish Rab-11a_frog Rab-11b_frog Rab-11a_chicken Rab-11b_chicken Rab-11a mouse Rab-11b_mouse Rab-11a_rat Rab-11b_rat Rab-11a_human Rab-11b human

Consensus Rab-11.1_C.elegans 8 Rab-11.2_C.elegans 78 Rab-11_fruitfly Rab-11a_medaka Rab-11b_medaka Rab-11b' medaka Rab-11a_zebrafish Rab-11ba_zebrafish Rab-11bb_zebrafish Rab-11a_frog Rab-11b frog Rab-11a chicken Rab-11b_chicken Rab-11a_mouse Rab-11b_mouse Rab-11a rat Rab-11b_rat Rab-11a_human Rab-11b_human

Consensus

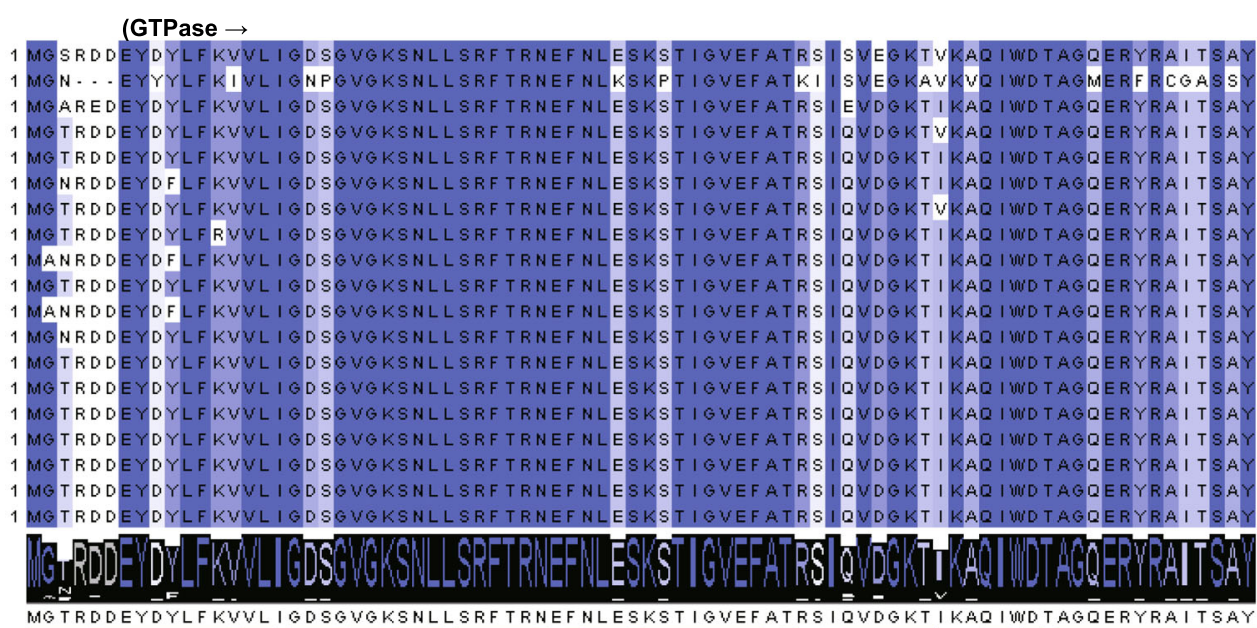

MGTRDDEYDYLF KVVL IGDSGVGKSNLLSRFTRNEFNLESKSTIGVEFATRSIQVDGKT I KADIWDTAGQERYRAITSAY

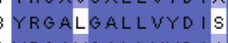

1 YRGAVGALLVYDIA

1 YRGAVGALLVYDIA

1 YRGAVGALLVYD I A

YRGAVGALLVYDIA

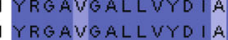

1 YRGAUGALL WYD I A

81 YRGAVGALLVYDIA

81 YRGAVGALLVYD I AK

1 YRGAVGALLVYDIAK

1 YRGAVGALLVYDIAK

1 YRGAVGALLVYDIAK

1 YRGAVGALLVYDIAKHL

1 YRGAVGALLVYD IAK

81 YRGAUGALLVYD I AKH
81 YRGAVGALLVYD I AKH

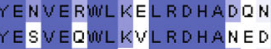

YENUER WLLELLRDADON

YENVERWL KELRDHADSN

YENVERWLKELRDHADSN

ENVERMLKELRDHADSN

YENVERWLKELRDHADSN

EN VERUL KELRDHADNN
EN WERULLELRDHADNN

EN SERULKELRDHADNK

YENVERIOLLKLRDHADNN

ENVERWLKELRDHADSN

EN VERWL KELRDHADNN

YENVER WL KELRDHADSN

TYN

ENUERUIL KELRDHADSN

YENUERWL KELRDHADSN
YENUERMLKKLRDHADSN

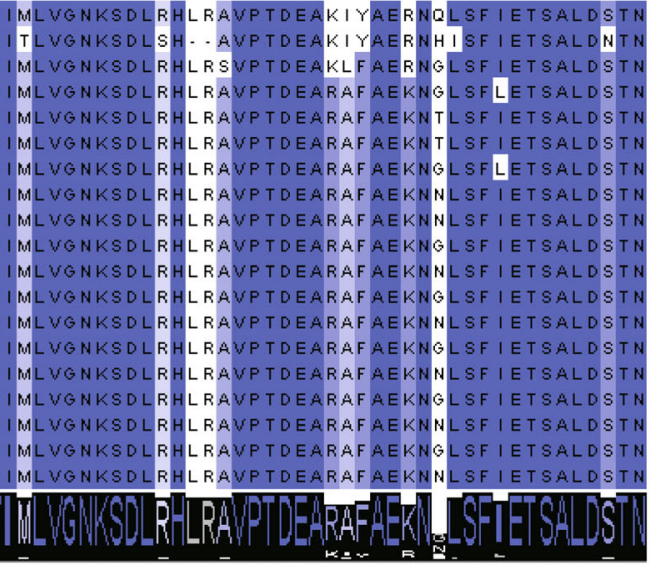

YRGAVGALLVYDIAKHLTYENVERIOLKELRDHADSNIVIMLVGNKSDLRHLRAVPTDEARAFAEKNGLSFIETSALDSTN

Rab-11.1_C.elegans 161 VEAAFTNILTE I YKSVSNKHVGTDRQGYGGGSGTIIPS...PASDPPK... KQCCIP.

Rab-11.2_C.elegans 156 VEAAF TN I VT E I YKLVSEKYKDHSG . . . . . . TIIPSTASNTP . KNQCCFP.

Rab-11_fruitfly 161 VETAFQNILTEIYR IVSQKQIRDPFEGDVIRFSNVEFIDVKPTVTADV - RKQCCO.

Rab-11a_medaka 161 VETAFQTI LTE I YR I VSQKOMSERQESDMSPSNNWUN I QVPF. TENKF - K KMQCCQNI

Rab-11b medaka 151 VEEAFKNILTEIYRIVSQRQIADRSAHDDSPGNNVUDISVPFTMDGQRGNKLPCCQSP

Rab-11b'_medaka 161 VEEAF KNVLTE I YR I VSQKQIADRSAHDESPGNNWVDISVPPT SDGQKGNKIQCCQSP

Rab-11a_zebrafish 161 VETAFQTILTE I YR I VSQKQMSDRRDNDMSPSNNVUSIQVQP. TENKP. - KMQCCQS I

Rab-11ba_zebrafish 181 VEEAF KN I L TE I YR I VSQKQMUDRPGHDESPGNNUUD I SUPPT T DGLKGNKFQCCQNL

Rab-11bb_zebrafish 161 VEEAF KN I L TE I YR I VSOKD I AER SAHDESPGNNUUD I SVPPT T DGDKSNKLOCCONL

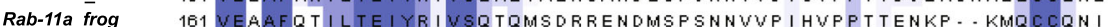

Rab-11b_frog 161 VEEAFKNILTEIYRIVSOKQIAERSAHGESPGNNVUDISVPPTTDGQKSNKLQCCQNL

Rab-11a_chicken 161 VEAAFQT I L TE I YR I VSQKQMSDRRENDMSPSNNUVP I HVPPT TENKP. . KMOCCQN I

Rab-11b_chicken 161 VEEAFKN I L TE I YR I VSQKQIADRSAHDESPGNNVUD I SVPFTTDGQKSNKLQCCQNL

Rab-11a_mouse 151 VEAAFQTILTE I YR IVSOKQMSDRRENDMSPSNNWUP I HVPFT TENKF . . KVOCCQN I

Rab-11b mouse 151 VEEAF KNILTEIYR IVSQKQIADRAAHDESPGNNVVDISVFPT TDGQRPNKLQCCQSL

Rab-11a_rat 161 VEAAFQTILTE I YR IVSQKQMSDRRENDMSPSNNWUPIHVPPTTENKP - KVQCCQN I

Rab-11b_rat 161 VEEAFKN I L TE I YR IVSOKQIADRAAHDESPGNNUVDISVPPT TDGQKPNKLQCCQNL

Rab-11a_human 181 VEAAFQT I LTE I YR I VSQKQMSDRRENDMSPSNNUVPI HVPPT TENKP - KVQCCQNI

Rab-11b human

Consensus

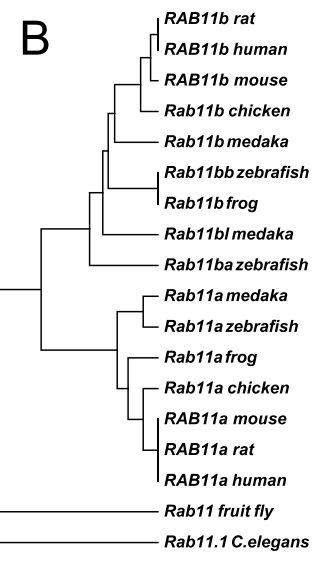

Rab11.2C.

Fig. 1 (See legend on next page.) 
(See figure on previous page.)

Fig. 1 Rab11 is highly conserved during evolution. a Alignment of Rab11 protein amino acid residue sequences of Caenorhabditis elegans, Danio rerio, Oryzias latipes, Xenopus tropicalis, Gallus gallus, Mus musculus, Rattus norvegicus and Homo sapiens. The sequences accessions IDs are listed following respectively, NP_490675, NP_001251691, NP_599137, NP_477170, XP_004086096, XP_004068250, XP_004068248, NP_001016481, NP_001120303, NP_001005827, NP_001012569, NP_059078, NP_033023, NP_112414, NP_116006, NP_004654, NP_004209. These protein sequences were aligned using ClustalW2 program and edited by Jalview. The GTPase domain sequence is indicated. b Phylogenetic tree of amino acid sequences generated using the MEGA6 software

brain, retina, and spinal cord except for the caudal part and the proctodeum from $20 \mathrm{hpf}$ to $24 \mathrm{hpf}$ (Fig. 5B, C). Its expression in the spinal cord turned weaker at $36 \mathrm{hpf}$ (Fig. 5D, D'). At $48 \mathrm{hpf}$ and $72 \mathrm{hpf}, \mathrm{rab11bb}$ expression was restricted to the brain, with expression in the retina and ear weakly detected and no detectable expression in other tissues (Fig. 5E-F'). Similar to rab11a and rab11ba, rab11bb was also observed to express in the brain at $48 \mathrm{hpf}$ but with a seemingly lower level by comparing the hybridization signals (Fig. $5 \mathrm{H}-\mathrm{H}^{\prime \prime}$ ). At $4 \mathrm{dpf}$, its expression in the brain remained strong, while its mRNA was also detected in the retina, ear and pharyngeal arches (Fig. 5G, G').

\section{Discussion}

Here we described the developmental expression patterns of rab11a, rab11ba and rab11bb. So far, although it has been reported that rab11a is expressed in epithelia [14] and myoblasts [15] in fruit fly, and in various tissues in mouse and human [16], the developmental expression of these genes in a whole organism was not described. It was also reported that Rab11a and Rab11b were localized to distinct cellular compartments in mouse [9]. The different expression patterns indicate the rab11 genes play diverse roles during development. In recent years, studies have proved the functional relationships between rab11 family proteins and neuron system development [17]. For instances, rab11 was reported to be a regulator of presynaptic function and axon regeneration [18, 19], Rab11-dependent recycling pathway was revealed to be involved in regulating cortical neuronal migration via the regulation of N-cadherin trafficking [20, 21]. in Drosophila, rab11 was found to regulate dendrite pruning of sensory neurons via regulating degradation of Neuroglian [22].

In this study, we found all the three rab11 paralogues were expressed in the brain from $24 \mathrm{hpf}$ to $72 \mathrm{hpf}$, suggesting their essential function during brain development and neural development. However, the three rab11 genes also showed differences in their expression patterns which implied distinct functions of them. For, example, we found rab11ba rather than the other two rab genes was highly expressed in cranio-facial neuromasts. In neuromasts, hair cells sense stimuli from the outside with their apical cilia, whose development and function are regulated by rab11ba [7]. Therefore, rab11ba might be essential for the development and function of hair cells in zebrafish. Besides, rab11a and rab11ba were also found to express in caudal vein at around $24 \mathrm{hpf}$, suggesting their potential regulating roles in vascular development of zebrafish embryos. More clues to the functional differentiation of the three rab genes need to be obtained in future studies.

\section{Conclusions}

There are three rab11 genes named rab11a, rab11ba and $r a b 11 b b$ in zebrafish. In this study, we performed the phylogenetic analysis of these Rab11 proteins of zebrafish and found they are highly conserved among diverse animal species. Further investigations using RT-PCR and in situ hybridization demonstrated the expression patterns of rab11 genes during zebrafish embryonic development. The results indicated that these rab11 genes might play

\begin{tabular}{|c|c|c|}
\hline & $1-\mathrm{c} \quad 64-\mathrm{c} \quad 50 \% \quad 12 \mathrm{~h} \quad 24 \mathrm{~h} \quad 30 \mathrm{~h} \quad 36 \mathrm{~h} \quad 48 \mathrm{~h} \quad 72 \mathrm{~h} \quad 96 \mathrm{~h}$ & heart kidney eye fin brain ovary skin muscle cord \\
\hline rabl1a & -------- & $-----\infty-$ \\
\hline efla & 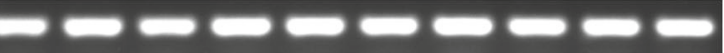 & ک \\
\hline rabl1ba & $---\infty-1$ & $\cdots--\infty-\infty$ \\
\hline efla & ת & ל \\
\hline$r a b 11 b b$ & $---\pi-$ & ------ \\
\hline efla & 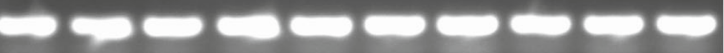 & ( \\
\hline
\end{tabular}



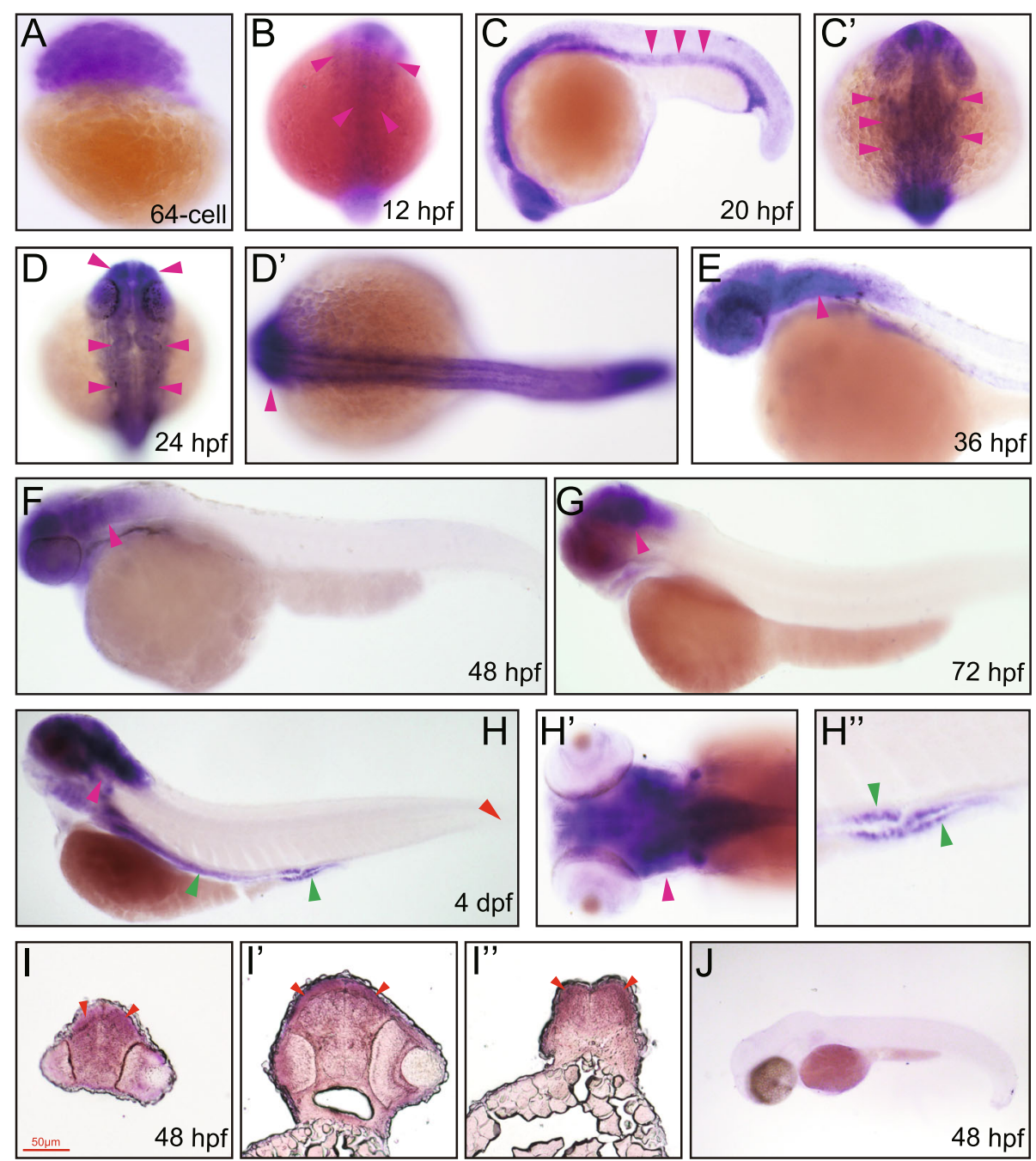

Fig. 3 Whole mount in situ and transverse section hybridization analysis of rab11a in zebrafish embryos. A 64 cell, lateral view. B 12 hpf, dorsal view, red arrowheads indicate the ubiquitous expression. C 20 hpf, lateral view, red arrowheads indicate endoderm. $\mathbf{C}^{\prime} 20$ hpf, dorsal view, red arrowheads indicate brain. D 24 hpf, dorsal view, red arrowheads indicate brain. D' 24 hpf, dorsal view, red arrowheads indicate hindbrain. E 36 hpf, lateral view, red arrowhead indicates brain. F 48 hpf, lateral view, red arrowhead indicates brain. G 72 hpf, lateral view, red arrowhead indicates brain. $\mathbf{H} 4$ dpf, lateral view, red arrowhead indicates brain; green arrowheads indicate pronephric duct. $\mathbf{H}^{\prime} 4$ dpf, dorsal view, arrowhead indicates brain. $\mathbf{H}^{\prime \prime} 4 \mathrm{dpf}$, lateral view, green arrowheads indicate pronephric duct. I 48 hpf, the trunk transverse section of the embryonic forebrain, arrowheads indicate cerebral cortex. I' $48 \mathrm{hpf}$, the trunk transverse section of the embryonic midbrain, arrowheads indicate cerebral cortex. I" 48 hpf, the trunk transverse section of the embryonic hindbrain, arrowheads indicate cerebral cortex. J 48 hpf, lateral view, embryos stained with the sense rablla probe

vital roles during nervous system development. These findings could provide new evidences for better understanding the functions of rab11 in the development of zebrafish embryos.

\section{Methods}

\section{Zebrafish tissue and embryos}

The zebrafish (AB line) in this study was obtained from China Zebrafish Resource Centre and maintained in Jiangsu key laboratory of neuroregeneration of Nantong university. Animal experiments were conducted conforming to the local institutional laws and the Chinese law for the Protection of Animals. Embryos were obtained through natural mating ( $\mathrm{AB}$ line) and maintained at $28.5^{\circ} \mathrm{C}$. Embryonic stages are as described [23]. Embryos after $24 \mathrm{hpf}$ were treated with $0.2 \mathrm{mM}$ 1-phenyl-2-thio-urea (PTU). Zebrafish embryos were collected at various stages, fixed with $4 \%$ paraformaldehyde (PFA) in phosphate-buffered saline (PBS) overnight at $4{ }^{\circ} \mathrm{C}$ or $2 \mathrm{~h}$ at room temperature, washed with PBST, dehydrated in methanol and stored at $-20^{\circ} \mathrm{C}$ until use. Embryos younger than $24 \mathrm{hpf}$ were dechorionated after fixation, prior to storage. 

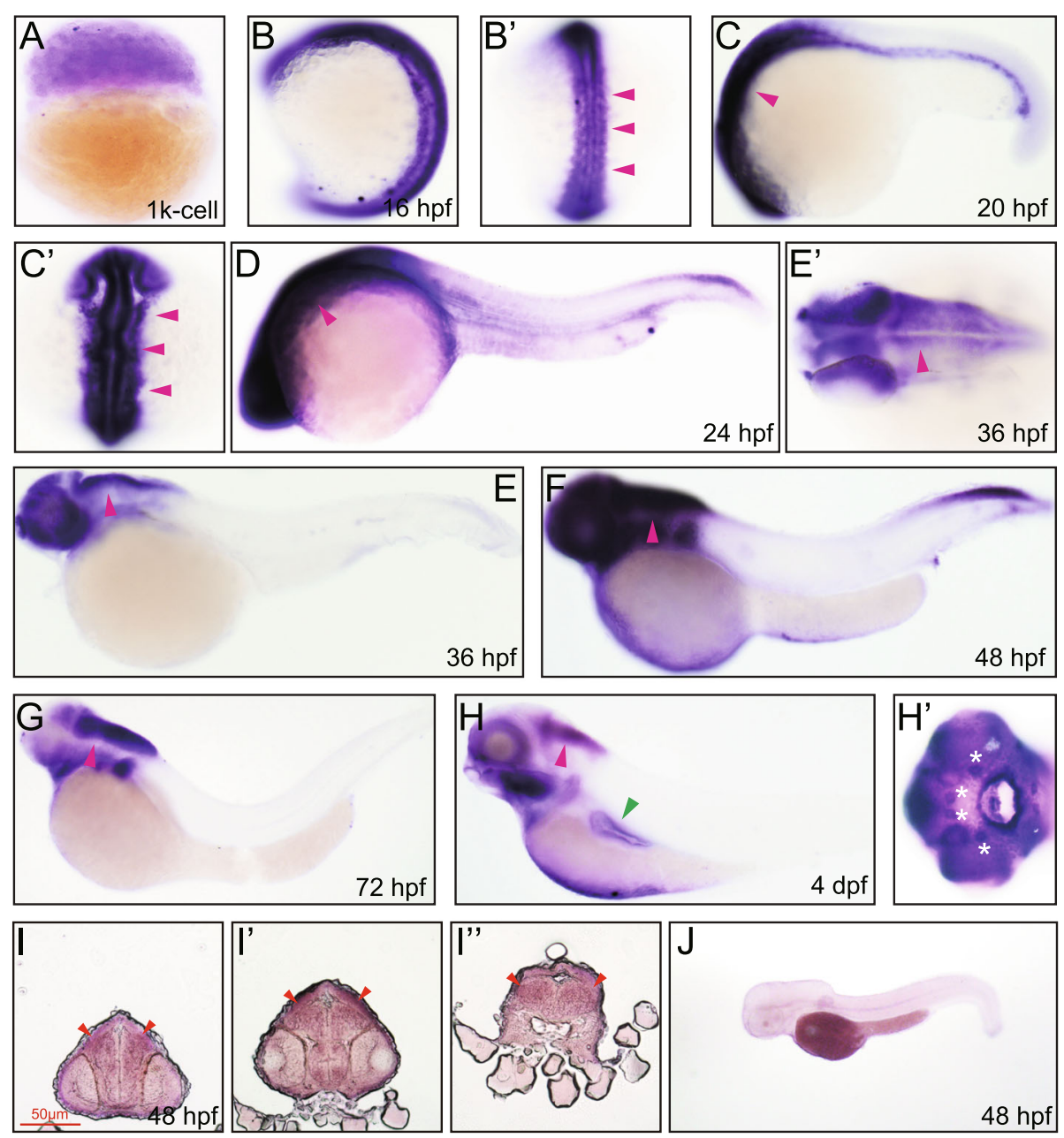

Fig. 4 Whole mount in situ and transverse section hybridization analysis of rab11ba in zebrafish embryos. A $1 \mathrm{k}$ cell, lateral view. B 16 hpf, lateral view. B' 16 hpf, dorsal view, arrowheads indicate neural tube. C 20 hpf, lateral view, arrowhead indicates brain. C' 20 hpf, dorsal view, arrowheads indicate brain. D 24 hpf, lateral view, arrowhead indicate brain. E 36 hpf, lateral view, arrowhead indicates spinal cord. E' 36 hpf, dorsal view, arrowhead indicates brain and head structure. F 48 hpf, lateral view, arrowhead indicates spinal cord. G $72 \mathrm{hpf}$, lateral view, arrowhead indicates brain. $\mathbf{H} 4 \mathrm{dpf}$, lateral view, red arrowhead indicates brain, green arrowhead indicates pectoral fins. $\mathbf{H}^{\prime} 4 \mathrm{dpf}$, asterisks indicate neuromasts. $\mathbf{I} 48$ hpf, the trunk transverse section of the embryonic forebrain, arrowheads indicate cerebral cortex. I' 48 hpf, the trunk transverse section of the embryonic midbrain, arrowheads indicate cerebral cortex. I" 48 hpf, the trunk transverse section of the embryonic hindbrain, arrowheads indicate cerebral cortex. J 48 hpf, lateral view, embryos stained with the sense rab11ba probe

\section{Bioinformatics}

The zebrafish rab11a, rab11ba and rab11bb sequence and conserved domains information was got form Ensembl (rab11a: ENSDARG00000041450, rab11ba: ENSDARG00000041878, rab11bb: ENSDARG000000900 86). Rab11 sequences were aligned by the ClustalW2 program (http://www.ebi.ac.uk/Tools/msa/clustalw2/). And the phylogenetic tree was built by MEGA6 software. Primers for PCR were designed by the Primer Premier 5 software.

\section{RNA extraction, reverse transcription, and RT-PCR}

Tissue was homogenized and frozen in TRIzol Reagent (Invitrogen) and stored at $-80^{\circ} \mathrm{C}$. The RNA was extracted following the manufacturer's instruction. $1 \mu \mathrm{g}$ of RNA was reverse transcribed into cDNA by the use of Transcriptor First Strand cDNA Synthesis Kit (Roche) according to the manufacturer's instructions. Synthesized cDNA was stored at $-20^{\circ} \mathrm{C}$. The primers for RT-PCR are listed, for efla: left primer ef1a-1-L TGATCTACAAATGCGGTGGA, right primer efla-1-R CAATGGTGATACCACGCTCA (141 bp); for rab11a: left primer, AAGCCGTTAGCATGGCTACT; right primer, CTTTTATTGCCCACCAGCAT (502 bp); for rab11ba: left primer, CAGGACAGGAACGCTACAGA; right primer, CACAACACACAGGAGCGAAA (540 bp); for rab11bb: left primer, CTGCTGTCGAGATTCACACG; Right primer, CGTGGTTACAGGTTTTGGCA, (584bp). 

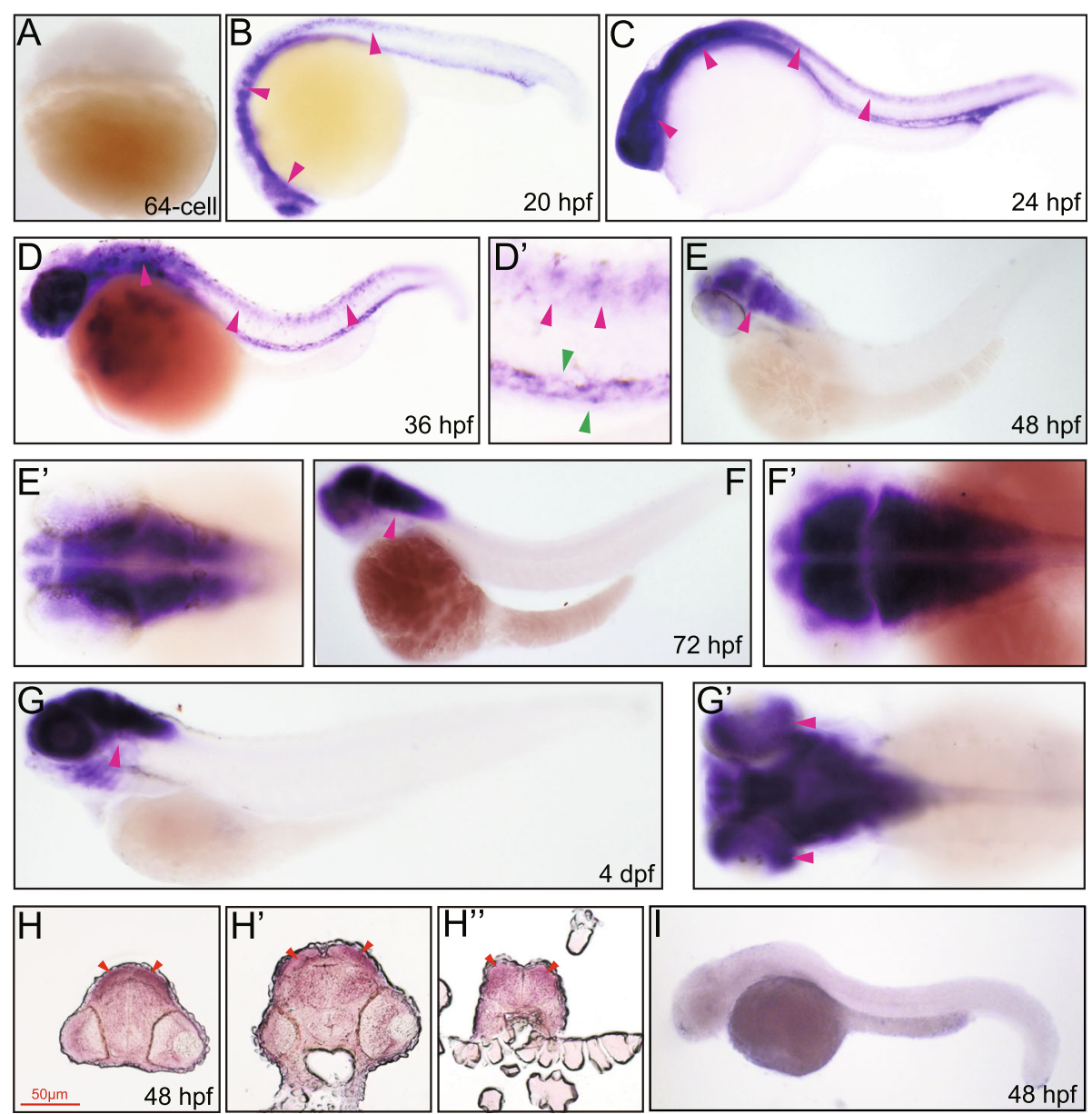

Fig. 5 Whole mount in situ and transverse section hybridization analysis of rab11bb in zebrafish embryos. A 64 cell, lateral view. B 20 hpf, lateral view, arrowheads indicate brain and spinal cord. C 24 hpf, lateral view, arrowheads indicate brain and spinal cord. D 36 hpf, lateral view, arrowheads indicate spinal cord. D' 36 hpf, dorsal view, red arrowheads indicate spinal cord, green arrowheads indicate pronephric duct. E 48 hpf, lateral view, arrowhead indicates brain. E' 48 hpf, dorsal view. F 72 hpf, lateral view, arrowhead indicates ear. F' 72 hpf, dorsal view. G 4 dpf, lateral view, arrowhead indicates pharyngeal arches. G' 96 hpf, dorsal view, arrowhead indicates retina. $\mathbf{H} 48$ hpf, the trunk transverse section of the embryonic forebrain, arrowheads indicate cerebral cortex. $\mathbf{H}^{\prime} 48 \mathrm{hpf}$, the trunk transverse section of the embryonic midbrain, arrowheads indicate cerebral cortex. $\mathbf{H}^{\prime \prime} 48$ hpf, the trunk transverse section of the embryonic hindbrain, arrowheads indicate cerebral cortex. I 48 hpf, lateral view, embryos stained with the sense rab11bb probe

All PCR amplifications were carried out in a total volume of $50 \mu \mathrm{l}$ using specific primers and Advantage2 Polymerase Kit (Clontech).

\section{Whole mount in situ hybridization and sectioned histological analysis}

Rab11a, rab11ba and rab11bb cDNA fragments were cloned into pGEM-T easy vector (Promega) with primers described in Section 2.3. RNA extraction, reverse transcription, and RT-PCR. Digoxigenin (DIG)-labeled RNA sense and antisense probes were made from the linearized plasmids according to the manufacturer's protocol using the DIG RNA Labeling Kit (SP6/T7) (Roche). The procedure was modified from Thisse in situ hybridization protocol [24]. The small baskets were not used in our protocol. BM purple AP substrate (Roche) was used instead of the staining solution. We use the BBR (Boehringer blocking reagent, Roche) for blocking.

For sectioned histological analysis, the whole-mount in situ hybridized embryos were fixed in 4\% PFA for $2 \mathrm{~h}$ at room temperature and then washed with PBST three times for $5 \mathrm{~min}$ each to remove the fixative reagents. The procedure was followed by a two-step embedment: one with $1.5 \%$ agarose $-5 \%$ sucrose solution for adjusting the fish orientation, and the other with Tissue-Tek OCT compound (SAKURA). The embryos were then sectioned with a Leica RM2125 microtome at $12 \mu \mathrm{m}$. After drying for $4 \mathrm{~h}$ at $37^{\circ} \mathrm{C}$, the sectioned samples were washed again with PBS and mounted and coverslipped with mounting medium. Pictures were taken with an Olympus DP70 camera on an Olympus stereomicroscope MVX10. 


\section{Abbreviations}

BBR: Boehringer blocking reagent; DIG: Digoxigenin; dpf: Days post fertilization; hpf: Hours post fertilization; PBS: Phosphate-buffered saline; PBST: Phosphate-buffered saline tween-20; PFA: Paraformaldehyde; PTU: 1phenyl-2-thio-urea; RT-PCR: Reverse transcription polymerase chain reaction

\section{Acknowledgements}

Not applicable.

\section{Authors' contributions}

ZH performed the experiments and analyzed the data, YG analyzed the data and performed the imaging, PPQ performed the experiments and analyzed the data, ZD provided useful discussion points and wrote part of the manuscript, WH contributed to manuscript proofreading, DL contributed to project planning and manuscript writing and proofreading, XD contributed to project planning, analyzed data and wrote the manuscript. All authors read and approved the final manuscript.

\section{Funding}

This work was supported by National Natural Science Foundation of China (81870359; 81570447; 81701222), Natural Science Foundation of Jiangsu Province (BK20180048, 17KJA180008), Natural Science Foundation of the Jiangsu Higher Education Institutions (18KJB180023), Science and Technology Project of Nantong (JC2018015, MS12017015-6) and the Research Innovation Program for College Graduates of Jiangsu Province (KYCX17_1909). The funding bodies had no role in the study design, data collection and analysis, or writing of the manuscript.

\section{Availability of data and materials}

All data generated and analyzed during this study are included in this published acticle.

\section{Ethics approval and consent to participate}

All animal experimentation was carried out in accordance with the NIH Guidelines for the care and use of laboratory animals (http://oacu.od.nih.gov/ regs/index.htm) and ethically approved by the Administration Committee of Experimental Animals, Jiangsu Province, China [Approval ID: SYXK (SU) 20070021].

\section{Consent for publication}

Not applicable.

\section{Competing interests}

The authors declare that they have no competing interests.

\section{Author details}

${ }^{1}$ Co-innovation Center of Neuroregeneration, Jiangsu Key Laboratory of Neuroregeneration, Nantong University, 19\# Qixiu Road, Nantong 226001 China. ${ }^{2}$ College of life science, Nantong University, 9\# Seyuan Road, Nantong 226001, China

\section{Received: 30 June 2019 Accepted: 11 December 2019}

\section{0.}

\section{References}

1. Novick P, Zerial M. The diversity of Rab proteins in vesicle transport. Curr Opin Cell Biol. 1997;9(4):496-504.

2. Schimmöller F, Simon I, Pfeffer SR. Rab GTPases, directors of vesicle docking. J Biol Chem. 1998:273(35):22161-4.

3. Chavrier P, Parton RG, Hauri HP, Simons K, Zerial M. Localization of low molecular weight GTP binding proteins to exocytic and endocytic compartments. Cell. 1990;62(2):317-29.

4. Sakurada K, Uchida K, Yamaguchi K, Aisaka K, Ito S, Ohmori T, Takeyama Y, Ueda T, Hori Y, Ohyanagi H, et al. Molecular cloning and characterization of a ras p21-like GTP-binding protein (24KG) from rat liver. Biochem Biophys Res Commun. 1991;177(3):1224-32.

5. Knodler A, Feng SS, Zhang J, Zhang XY, Das A, Peranen J, Guo W. Coordination of Rab8 and Rab11 in primary ciliogenesis. Proc Natl Acad Sci U S A. 2010;107(14):6346-51.

6. Ullrich $\mathrm{O}$, Reinsch $\mathrm{S}$, Urbe $\mathrm{S}$, Zerial M, Parton RG. Rab11 regulates recycling through the pericentriolar recycling endosome. J Cell Biol. 1996;135(4):913-24.
7. Westlake CJ, Baye LM, Nachury MV, Wright KJ, Ervin KE, Phu L, Chalouni C, Beck JS, Kirkpatrick DS, Slusarski DC, et al. Primary cilia membrane assembly is initiated by Rab11 and transport protein particle II (TRAPPII) complexdependent trafficking of Rabin8 to the centrosome. Proc Natl Acad Sci U S A. 2011;108(7):2759-64.

8. Bhartur SG, Calhoun BC, Woodrum J, Kurkjian J, lyer S, Lai F, Goldenring JR. Genomic structure of murine Rab11 family members. Biochem Biophys Res Commun. 2000;269(2):611-7.

9. Lapierre LA, Dorn MC, Zimmerman CF, Navarre J, Burnette JO, Goldenring JR. Rab11b resides in a vesicular compartment distinct from Rab11a in parietal cells and other epithelial cells. Exp Cell Res. 2003;290(2):322-31.

10. Haugsten EM, Brech A, Liestøl K, Norman JC, Wesche J. Photoactivation approaches reveal a role for Rab11 in FGFR4 recycling and Signalling. Traffic. 2014;15(6):665-83.

11. Grimsey NJ, Coronel LJ, Cordova IC, Trejo J. Recycling and endosomal sorting of protease-activated receptor-1 is distinctly regulated by Rab11A and Rab11B proteins. J Biol Chem. 2016;291(5):2223-36.

12. Zulkefli KL, Houghton FJ, Gosavi P, Gleeson PA. A role for Rab11 in the homeostasis of the endosome-lysosomal pathway. Exp Cell Res. 2019;380(1):55-68.

13. Clark BS, Winter MR, Cohen AR, Link BA. Generation of Rab-based transgenic lines for in vivo studies of endosome biology in zebrafish. Dev Dyn. 2011; 240(11):2452-65.

14. Sasikumar S, Roy JK. Developmental expression of Rab11, a small GTPbinding protein in Drosophila epithelia. Genesis. 2009;47(1):32-9.

15. Bhuin T, Roy J. Rab11 is required for myoblast fusion in Drosophila. Cell Tissue Res. 2009;336(3):489-99.

16. Su Al, Wiltshire T, Batalov S, Lapp H, Ching KA, Block D, Zhang J, Soden R, Hayakawa M, Kreiman G, et al. A gene atlas of the mouse and human proteinencoding transcriptomes. Proc Natl Acad Sci U S A. 2004;101(16):6062-7.

17. Shikanai M, Yuzaki M, Kawauchi T. Rab family small GTPases-mediated regulation of intracellular logistics in neural development. Histol Histopathol. 2018;33(8):765-71.

18. Nieuwenhuis B, Eva R. ARF6 and Rab11 as intrinsic regulators of axon regeneration. Small GTPases. 2018:1-10.

19. Kokotos AC, Peltier J, Davenport EC, Trost M, Cousin MA. Activity-dependent bulk endocytosis proteome reveals a key presynaptic role for the monomeric GTPase Rab11. Proc Natl Acad Sci U S A. 2018;115(43):E10177-86.

20. Barnat M, Le Friec J, Benstaali C, Humbert S. Huntingtin-mediated multipolar-bipolar transition of newborn cortical neurons is critical for their postnatal neuronal morphology. Neuron. 2017;93(1):99-114.

21. Kawauchi T, Sekine K, Shikanai M, Chihama K, Tomita K, Kubo K-i, Nakajima K, Nabeshima Y-i, Hoshino M. Rab GTPases-dependent endocytic pathways regulate neuronal migration and maturation through $\mathrm{N}$-cadherin trafficking. Neuron. 2010;67(4):588-602.

22. Krämer R, Rode $S$, Rumpf $S$. Rab11 is required for neurite pruning and developmental membrane protein degradation in Drosophila sensory neurons. Dev Biol. 2019;451(1):68-78.

23. Kimmel CB, Ballard WW, Kimmel SR, Ullmann B, Schilling TF. Stages of embryonic development of the zebrafish. Dev Dyn. 1995;203(3):253-310

24. Thisse C, Thisse B. High-resolution in situ hybridization to whole-mount zebrafish embryos. Nat Protoc. 2008;3(1):59-69.

\section{Publisher's Note}

Springer Nature remains neutral with regard to jurisdictional claims in published maps and institutional affiliations.

Ready to submit your research? Choose BMC and benefit from:

- fast, convenient online submission

- thorough peer review by experienced researchers in your field

- rapid publication on acceptance

- support for research data, including large and complex data types

- gold Open Access which fosters wider collaboration and increased citations

- maximum visibility for your research: over $100 \mathrm{M}$ website views per year

At $\mathrm{BMC}$, research is always in progress.

Learn more biomedcentral.com/submissions 